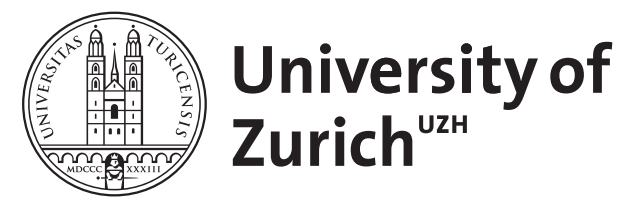

\title{
Toward an embodied cognitive semantics
}

\author{
Stocker, Kurt
}

\begin{abstract}
This article strives to move forward toward an embodied account of cognitive semantics. Using current empirical embodiment findings and theoretical interpretations thereof, the major schematic systems of Talmyan cognitive semantics (configurational structure, attention, perspective, and force dynamics) are all presented within one unified embodied account. It will be shown that there is much empirical evidence that thought is spatialized and that our eyes project this spatialized thought into the external (usually near) space in front of our body. This - eyes projecting spatialized thought into peripersonal space - will be called the theory of ocular cognitive semantics (and will sometimes also more generally be referred to as the theory of the ocular mind). Ocular cognitive semantics is meant to be a first step forward toward a more comprehensive theory of embodied cognitive semantics. Clinical implications of ocular cognitive semantics for people with schizophrenia are discussed.
\end{abstract}

DOI: https://doi.org/10.1163/23526416-00102002

Posted at the Zurich Open Repository and Archive, University of Zurich

ZORA URL: https://doi.org/10.5167/uzh-119541

Journal Article

Accepted Version

Originally published at:

Stocker, Kurt (2015). Toward an embodied cognitive semantics. Cognitive Semantics, 1(2):178-212.

DOI: https://doi.org/10.1163/23526416-00102002 


\title{
Toward an embodied cognitive semantics
}

\author{
Kurt Stocker \\ Department of Psychology \\ University of Zurich \\ kurt.stocker@psychologie.uzh.ch
}

\begin{abstract}
This article strives to move forward toward an embodied account of cognitive semantics. Using current empirical embodiment findings and theoretical interpretations thereof, the major schematic systems of Talmyan cognitive semantics (configurational structure, attention, perspective, and force dynamics) are all presented within one unified embodied account. It will be shown that there is much empirical evidence that thought is spatialized and that our eyes project this spatialized thought into the external (usually near) space in front of our body. Thiseyes projecting spatialized thought into peripersonal space-will be called the theory of ocular cognitive semantics (and will sometimes also more generally be referred to as the theory of the ocular mind). Ocular cognitive semantics is meant to be a first step forward toward a more comprehensive theory of embodied cognitive semantics. Clinical implications of ocular cognitive semantics for people with schizophrenia are discussed.
\end{abstract}

Keywords: cognitive semantics, eye movements, mental time line, attention, field perspective, observer perspective, force dynamics, schizophrenia

\section{Introduction}

Talmy calls his vast body of work on the nature of human cognition and language "cognitive semantics" and characterizes its basics in the following way:

... the word "semantic" simply refers to the specifically linguistic form of the more generic notion "conceptual." Thus, general conception - that is, thought -includes linguistic meaning within its greater compass. . . research on cognitive semantics is research on conceptual content and its organization in language and, hence, on the nature of conceptual content in general. In this formulation, conceptual content is understood to encompass not just ideational content but any experiential content, including affect and perception (Talmy, 2000a, p. 4).

Thus, cognitive-semantic research is understood to include studying thought per se. This naturally brings a general psychological component to this line of research. Indeed, one of the main concerns of the cognitive-semantic approach - as well as of the cognitive-linguistic approach in general-has always been "to integrate the linguistic and the psychological perspectives on cognitive organization in a unified understanding of human conceptual structure" (Talmy, 2000a, p. 3). In this article I provide an account of such "a unified understanding of human conceptual structure" by showing that cognitive research is at a stage now that allows us to view cognitive semantics systematically through an embodied perspective. 
The definition of embodiment which I offer is that thought and emotion can be considered as embodied, when their cognitive processing is strongly and systematically influenced by the sensory and motor system. I think that this characterization of embodiment (sometimes without spelling it out explicitly) underlies much classic and current experimental work in the embodiment realm (e.g., Bergen \& Wheeler, 2009; Glenberg \& Kaschak, 2002; Hartmann, Martarelli, Mast, \& Stocker, 2014; Huette, Winter, Matlock, \& Spivey, 2014 ). Such a characterization of embodiment is also in line with one view on embodied cognition that has been described by Talmy $(2011,636-637)$, the view that "we do much of our thinking and reasoning in terms of. . . experientially derived structures" (p. 637). However, Talmy also puts a note of caution to this view, saying that if this view is applied too broadly, it "might obscure the possible existence of an actual cognitive system for objectivity and reason" (p. 637). Such a system might exist in a sui generis manner and might not be derived from sensory or motor experiences. I concur with this note of caution. In this context, I would like to stress that there is no principled reason which would allow us to assume the following: that the proposal that a thought is structured by the sensory and motor system and that the proposal that faculty of reasoning might also exist in a sui generis manner are contradictory. For instance, Knauff (2013) presents a large body of empirical evidence which shows convincingly that spatial representations — which he calls spatial layout models — might greatly facilitate logical reasoning. However, while these spatial layouts (which might well represent experientially derived structures) seem to greatly assist us in carrying out logical reasoning, the reason why logical reasoning exists in the first place can still not be merely explained by the existence of these spatial layouts (an observation which also seems to be in line with Knauff's views). Thus, when I in this article speak of "embodied cognitive semantics" I propose that cognitive-semantic concepts involve structures and processes of the sensory and motor systems-but at the same time, this does not preclude the possibility that cognitive systems other than the sensory and motor systems also contribute to the construction of thought-for instance thought systems such as logical reasoning.

My basic proposal in relation to embodied cognitive semantics is this: it is time to consider the possibility that much, if not all, of the cognitive processing that has been described in theory of cognitive semantics ((Talmy, 2000a,b, 2005, 2007a, 2011, 2015) is also systematically reflected in the physical eye. This proposal will lead to the theory of ocular cognitive semantics. As cognitive semantics is essentially a theory about the mind (Ibarretxe Antuñano, 2006, p. 253), I will sometimes also refer to the theory of ocular cognitive semantics simply as the theory of the "ocular mind." Thus these terms will be used interchangeably, depending on whether the focus is on cognitive-semantic theory or on the mind in general. Ocular cognitive semantics forms, as I hope to demonstrate, a substantial basic part of an embodied account of cognitive semantics. To provide an immediate first sense of how ocular phenomena can be theorized to relate to cognitive semantics, consider (1). (1) offers one major ocular (oculomotor or pupillometric) prediction in relation to the four major schematic systems of cognitive semantics (Talmy, 2000a): configurational structure, attention, perspective, and force dynamics. All of these predictions and the related empirical findings will be examined and discussed in the relevant sections.

(1) Ocular (oculomotor and pupillometric) predictions for major cognitive semantic schematic systems

a. Oculomotorically reflected configurational structure (related to the domain member notions 
"space" and "time”): Our eyes look at or along invisible geometric paths (parts of configurational structure) that are mentally projected into the peripersonal space in front of our body. For instance: our eyes look along an invisible mental time line in peripersonal space while mentally processing time (Section 2).

b. Oculomotorically reflected attention: Our eyes look more at foregrounded mental structure than at backgrounded mental structure in peripersonal space. For instance: our eyes look more at the temporal Figure point than at the temporal Ground point on the on the mental time line in peripersonal space (Section 4).

c. Oculomotorically reflected perspective: Our eyes look at mental structure in peripersonal space in a particular perspective. For instance: our eyes look upward to a future point on the mental time line in peripersonal space, or, as a possible perspectival alternative, our eyes look upward and rightward to a future point on the mental time line in peripersonal space (Section 3).

d. Pupillometrically reflected force dynamics: Our pupils might dilate more when cognitively processing more complex force-dynamic structure than when cognitively processing less complex force-dynamic structure. Additionally, force-dynamic structure can also be proposed to unfold along the mental time line in peripersonal space, for instance in relation to two specific points on the time line, one representing the cause and one representing the effect (Sections 7 and 8.2).

As I will show in this article, there is much empirical evidence that thought is spatialized and that our eyes project this spatialized thought into the external (usually near) space in front of our body. In the relevant experiments that will be discussed to support the theory of the ocular mind (ocularized cognitive semantics), this external space is often an empty (eye-tracked) screen. In more natural contexts, the external space might often be the peripersonal empty space right in front of us, roughly the same space that we sometimes also use for co-speech gestures. Thus, if one were to gesture, while, for example, verbally describing lower floors of a tall building, followed by a description of higher floors (without seeing the building), it would be natural to use gestures that would depict the upward progression of the description. However, such mental representations (of for example looking more and more upward along a tall building) are also "acted out" by corresponding upward eye movements, even when people are only imagining or recalling such scenes (cognitive activities which are not typically accompanied by manual gestures) (Spivey \& Geng, 2001). Likewise, the construction of a mental time line (for example, with the past to the left and the future to the right) cannot only be acted out by corresponding gestures while speaking (Casasanto \& Jasmin, 2012; Cooperrider \& Núñez, 2009), but can also be acted out by corresponding eye movements-for instance even when we only listen to (Stocker, Hartmann, Martarelli, \& Mast, in revision ) or imagine (Hartmann, Martarelli, Mast, \& Stocker, 2014) time-related concepts, instances where we typically do not manually gesture either. Perhaps then one way of thinking of the theory of the ocular mind is to say that "we gesture even if we don't gesture by hand"-because our eye movements "gesture" or "act out" these mental concepts into the external space in front of us, even in the absence of manual gestures. A kind of "gestural gaze" is projected by our eyes into the peripersonal space, and it is this gestural gaze that allows one to systematically bring in an embodied ocular dimension into cognitive semantics. That gaze can play a cognitively meaningful role has long been noticed in studies of social interaction, where specific uses of gaze for instance play a role in turn taking 
(Kendon, 1967; McNeill, 2006).

Based on decades of research, Talmy has characterized cognitive semantics as involving several cognitive schematic systems, the main ones being configurational structure, perspective, attention, and force dynamics (Talmy, 1988b, 2000a). While Talmy has laid out each of these schematic systems quite extensively, an account that synthesizes these different schematic systems into one larger unified whole has, to the best of my knowledge, not been provided yet. Hence, it has for instance not been laid out yet, how the Talmyan schematic system force dynamics (causal and causality-related thought) relates to the Talmyan schematic system configurational structure (which is mainly concerned with spatial and temporal thought). However, Talmy does provide a first basic sketch (a sketch which he does not follow up any further) how force-dynamic mental structure might manifest in spatiotemporal mental structure:

...force dynamics is a fourth schematic system: to the preceding basically pictorial complex [such as a mental image, which can be described with the configurational- structure, perspective, and the attention systems], one now adds the forces that the elements of the structural framework exert on each other (2000a, p. 467).

Given the theoretical components of the ocular mind theory-the cognitive components that I will use to describe configurational structure, attention, and perspective in Sections 2 to 7 Talmy's idea to "add the forces that the elements of the structural framework exert on each other" to the basically "pictorial complex" turns out to lead to rather complex mental representations. It will lead, as we will see, to a complex conglomerate of cognitive spacetime, perspective, and force dynamics. To introduce the new theory of the ocular mind in this article, I will mainly analyze and discuss the cognitive-semantic structure underlying the following sentence (from Stocker, 2014b, p. 129):

(2) Mary went to bed because she was tired.

This perhaps seemingly simple statement is of rich mental complexity and involves all of the main schematic categories that Talmy proposes for cognitive semantics: it involves configurational structure, attention, perspective (in both "space" and "time"), and force dynamics. In this article, I will examine the embodied (largely ocular) aspect of these cognitivesemantic schematic systems, exemplifying it with (2). One helpful start for teasing out the rich conceptual complexity of a seemingly simple statement like (2) is to consider the whole set of open-class and closed-class elements that such a sentence contains (cf. Talmy, 2000a, pp. 3334 ) -with the open-class specifications remaining a heuristic approximation. The open-class elements of (2) and what they specify are listed in (3):

(3) Open-class elements of Mary went to bed because she was tired ${ }^{1}$

A. Mary: occurring in "space," person, female, proper name, specified individual

${ }^{1}$ In some cases in (3) and (4) where "occurring in space" will be stated, the reader might wonder if rather not "occurring in time" should be stated—and vice versa. As an attempt to resolve these ambiguities, the concept of object spacetime for "space" and the concept of event spacetime for "time" (Stocker, 2014c) will be introduced in Section 2. 
(individuation), uniplex $^{2}$

B. go: occurring in "space," biped motion (for one possibility)

C. bed: occurr

flat piece of furniture intended for sleeping (for one possibility)

D. tired: occurring in "space," a physiological condition in which a considerable part of one's bodily or mental resources have been consumed

The cognitive-semantic schematic systems (the main concern of the present article) are mainly reflected in qualitative properties that the closed-class system of a language specifies (Talmy, 2000a, p. 40). Thus, the present article will focus on the schematic structuring that is revealed in (4), which lists the closed-class elements of (2) and what they specify. The cognitive semantic schematic systems (SYS) or schematic categories (CAT), to which the listed elements belong, are put in brackets.

(4) Closed-class elements of Mary went to bed because she was tired

\section{A. First use of past tense (for "go")}

1. occurring in "time" (SYS: configurational structure; CAT: domain)

2. an event (temporal Figure) occurs before the present moment (temporal Ground)

(SYS: attention; CAT: Figure and Ground $)^{3}$

3. temporal Figure and Ground can be schematized to points (no extension) on a mental time line (SYS: configurational structure; CAT: degree of extension)

B. Second use of past tense (past tense of "be" follows past tense of "go")

2 Plexity is a term from Talmy (2000a, pp. 48-50). It stands for the concepts of uniplexity and multiplexity, which, respectively, stand for "conceptual singularity" and "conceptual plurality" across both "space" and "time." Thus bird as well as sighed (once) can both be regarded as uniplex, and birds and kept sighing both as multiplex. Also certain forms that are grammatically speaking singular-e.g., furniture-are conceptually considered to be multiplex.

${ }^{3}$ Considered in its entirety, the cognitive-semantic system attention (Talmy, 2007a) is a huge cognitive system involved in assigning different degrees of salience to (often linguistically induced) cognitive representations. It can be broken up into some ten categories, most with subcategories, which contain over fifty to date identified basic attention mechanisms. Out of this huge range of cognitive attention mechanisms, for this article I chose to mainly focus on just two: the foundational notions of Figure and Ground. This choice was motivated because clear oculomotor (empirically confirmed) predictions can be made for temporal Figure and Ground on the mental time line (see Section 3). Thus, this is one first example of how attention phenomena are reflected in the physical eye. 
1. occurring in "time" (SYS: configurational structure; CAT: domain)

2. two events (temporal Composite Figure) occur before the present moment (temporal Ground of Composite Figure) (SYS: attention; CAT: Figure and Ground)

3. temporal Composite Figure and Ground of Composite Figure can be schematized to points (no extension) on a mental time line (SYS: configurational structure; CAT: degree of extension)

C. to

1. occurring in "space" (as one possibility) (SYS: configurational structure; CAT: domain)

2. motion toward an object (as one possibility) (SYS: configurational structure; CAT: path)

D. because temporal $_{\text {ber }}$

1. occurring in "time" (SYS: configurational structure; CAT: domain)

2. one event (cause) occurs before another event (effect) (for one temporal possibility of cause and effect occurrence) (SYS: attention; CAT: Figure and Ground)

E. because spatial/causal

1. occurring in "space" (SYS: configurational structure; CAT: domain) (in "space" since because assigns force-dynamic structure onto "objects" (including animate "objects") in "space"; cf. Section 2)

2. one entity (force-dynamic antagonist placed in the subordinate clause) imposes its force upon another entity (force-dynamic agonist placed in the main clause) (SYS: force dynamics)

F. she spatial

1. occurring in "space” (SYS: configurational structure; CAT: domain)

2. reference to an animate entity that possesses gender, as one possibility: person

a. uniplex (SYS: configurational structure; CAT: plexity)

b. female (SYS: configurational structure; CAT: gender)

c. other (as opposed to self): the speaker indicates that he/she is not the person referred to in the sentence (SYS: perspective; CAT: third person)

G. she temporal

1. occurring in "time" (discourse time) (SYS: configurational structure; CAT: domain) (in "time" because the anaphoric dimension of she refers to an "event" - a "speech event" in this case; cf. next point as well as Section 2) 
2. person last mentioned (reference back to the speech event, written event, etc. in which the person was last mentioned; anaphoric reference) (SYS: perspective; CAT: event)

In this way, in relation to (2) I will first examine: embodied domain (a most basic part of configurational structure) (Section 2), then add perspective in "time" to it (Section 3), then embodied attention (Section 4), to then further add embodied perspective in "space" (Section 5) as well as embodied plexity, gender, and path (further aspects of "spatial" configurational structure) (Section 6) - to then finally also "add the forces that the elements of the structural framework exert on each other" (Talmy, cf. above) (Section 7). Please note that (2) just serves exemplifying purposes. The proposal of the ocular mind (of ocular cognitive semantics) that can be developed in this manner can then be applied quite generally to thought.

Perhaps a note should be inserted here why "time" (Section 3) is addressed before "space" (Section 5). "Space" is still widely treated as more basic than "time" (e.g., Lakoff \& Johnson, 1999; Núñez \& Sweetser, 2006). However, other work-which we cannot examine in detail here-has in my view convincingly made a case that both "space" and "time" are equally basic (Evans, 2013; Galton, 2010; Stocker, 2014c; Talmy, 2011, pp. 636-637). Evans and Galton for instance argue convincingly that the concept of "transience" basically involves time; Talmy provides comparable arguments for temporal notions like "starting," "continuing", "stopping," "waiting," and others; and Stocker undertakes a detailed literature review (combined with cognitive-linguistic analysis) and concludes that there is "sui generis space" as well as "sui generis time" within human cognition. Thus, by first examining "time" (Section 3) and then "space" (Section 5), I do not want to imply that "time" is more basic than "space". Following the arguments of Stocker (2014c), I assume that both "space" and "time" exist in their own right. This, as also detailed in Stocker (2014c), does not exclude the additional possibility that "space" can be metaphorically mapped onto "time." The reason why I examine "time" before "space" in relation to (2), is that (2) in essence is a cause-and-effect structure, where the cause takes place first, and the effect second (called successful causation with consecutive cause and effect in Stocker (2014b)). Given this overall temporal structure, I find it more natural to examine "time" before "space" in this particular case.

\section{Embodied domain}

This section lays out the basic geometric pathways along which our eyes project mental content into the peripersonal space in front of us while we think about concepts that involve space and time. This allows us to lay out the geometric scaffoldings of the ocular mind. In Talmyan cognitive semantics, the notions of "space" and "time" relate to the foundational schematic category domain (which is part of the schematic system configurational structure).

The schematic category of domain has two principal member notions, 'space' and 'time'. . the kind of quantity existing in space is, generically, 'matter', and, in respectively continuous or discrete form, is 'mass' and 'objects'. The kind of quantity existing in time, is, generically, 'action' and, in continuous or discrete form, is 'activity' and 'acts' - terms here used neutrally as to whether the action is static or changing, autonomous or agentive (Talmy, 2000a, p. 42).

This space-time dichotomy, as for instance reflected in this quote of Talmy, is commonly found in psychology and cognitive science (Stocker, 2014c). However, in Stocker (2014c) I argued 
that we are now at a stage in cognitive science that allows us to go beyond the space-time dichotomy. Based on extensive linguistic analysis and experimental review, I developed two main proposals in that article. The first is that what is commonly referred to as "space" (or "spatial cognition") in the cognitive sciences always contains time. The second is that what is commonly referred to as "time" (or "temporal cognition") always contains space. Thus, I introduced the term object spacetime (or object-spatiotemporal cognition) for "space" (or "spatial cognition"), as well the term event spacetime (event-spatiotemporal cognition) for "time" (or "temporal cognition"). I also developed the terms in the same generic sense as in Talmy's quote above, keeping object and event spacetime for instance continuous/discreteneutral. Thus, I used the term "object" in a most generic sense. In cognitive spacetime, the conceptualized quantity in object spacetime is considered an "object", no matter if it is animate or inanimate, concrete or abstract, continuous or discrete. Likewise, the conceptualized quantity in event spacetime is considered an "event", no matter if it is continuous or discrete. Relating this analytic spacetime framework back to (4), everything that relates to an object in this generic sense is classified as "occurring in space" and everything that relates to an event in this generic sense is classified as "occurring in time"-which now translates into "occurring in object spacetime" and "occurring in event spacetime," respectively. In Stocker (2014c) I also tried to provide evidence that both object spacetime and event spacetime also consist of "sui generis space" and "sui generis time" (cf. 1) —with the additional possibility that event spacetime can also take on (at least in most languages/cultures) "space" that has been mapped from object spacetime.

For object spacetime, one of the main points that suggested proposing the existence of this subdomain was the argument that even static cognitive space-Talmy's stasis (2000b, p. 237) involves conceptualizing time. The proposal that even static space contains time was developed by using a specific cognitive-semantic method called "analytic manipulation" by Talmy (2007b). Such analysis gave evidence that even a static sentence like There are some houses in the valley contains time (see pp. 75-76 in Stocker, 2014c). For event spacetime, there were two main points which suggested proposing the existence of this subdomain. The first such observation was that temporal expressions which only etymologically relate to mapped space, expressions like before and after, can cognitive-linguistically be argued to contain specific spatial structure in their contemporary use - spatial structure that differs from their spatial structure in earlier days, when they still functioned as metaphors (Stocker, 2012a, 2014c). The second such observation is that the users of a language which seems largely to lack spatial metaphors for time (which seems a rare occurrence), nevertheless seem to mentally construe a spatial time line (as observed in Amondawa by Sinha, Sinha, Zinken, \& Sampaio, 2011).

Given their spatial component, both object and event spacetime consist of a certain mental geometry. Object spacetime consists of three-dimensional cognitive space $x y z$ with time $t$ unfolding along the fourth dimension as an abstract nonspatial correlate within the $x y z$-space (Stocker, 2014c; Fig. 1a). Take the example:

(5) One ball followed another down the spiral staircase.

Conceptualizing the mental scene underlying this sentence involves a mental representation where the balls move through the $x y z$-space of object spacetime (a spiral path in this case) and at the same time progress along fourth-dimensional $t$. Event spacetime consists (in most cultures/languages) of one-dimensional space $x$ with time $t$ unfolding as a correlate along the $x$ - 
path (Fig. 1b). The $x$-axis of event spacetime corresponds to what is often referred to as the mental time line in psychology and cognitive science (e.g., Arzy, Adi-Japha, \& Blanke, 2009; Hartmann \& Mast, 2012; Hartmann, Martarelli, Mast, \& Stocker, 2014; Ulrich \& Maienborn, 2010). Take the example (from Stocker, 2012a, p. 396):

(6) New Year's follows Christmas.

As examined in Stocker (2012a), conceptualizing the scene underlying this sentence, involves a mental representation where the events New Year's and Christmas are mentally construed as moving along the mental time line - or along $x t$-spacetime in the spatiotemporal terminology of Stocker (2014c). (The terms $x t$-spacetime and mental time line will be used interchangeably in this article). The mental time line will be further investigated in Section 3.

Fig. 1 illustrates and summarizes the proposed revision of the cognitive-semantic schematic category domain by laying out its basic geometric properties.

(a)

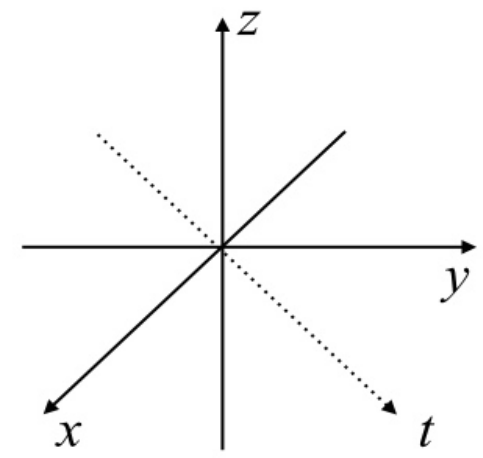

(b)

Figure 1: The cognitive-semantic schematic category domain (foundational part of the schematic system configurational structure) with its two principal member notions "space" and "time" (Talmy, 2000a: 42-47) has been, following Stocker (2014c), revised to in the current section to propose the domain cognitive spacetime with its two principal member notions (subdomains) a) object spacetime and b) event spacetime (Fig. from Stocker, 2014c, used with permission). These spatiotemporal structures function as geometric scaffoldings which are projected into the peripersonal space (in front of our body) by our gestural gaze.

In this section, we have developed the two principal member notions "space" and "time" of Talmy's cognitive-semantic schematic category domain (part of the system configurational structure) (Talmy, 2000a, pp. 42-47) into the new domain of cognitive spacetime with its two new principal member notions (subdomains) object spacetime and event spacetime (Stocker, 2014c). As has been discussed, the concept of cognitive spacetime can account for the observation that there is always "time" in what is commonly referred to as "space" in cognitive science and psychology, and there is always "space" in what is commonly referred to as "time" in cognitive science and psychology. Thus, all the cognitive-semantic elements that have been referred to as "occurring in space" in (3) and (4) can be more adequately thought of as "occurring in object spacetime," and all the cognitive-semantic elements that have been referred to as "occurring in time" can be more adequately thought of as "occurring in event spacetime."

Note that the domain of spacetime is so basic that not a single element in (3) and (4) is not either part of object spacetime or event spacetime. As we will see, in the theory of ocular cognitive semantics, such spatiotemporal properties are projected out by our gestural gaze into the 
peripersonal space in front of our body.

\section{Embodied perspective and configurational structure in "time"}

Having laid out the basic geometric properties of the configurational-structural schematic category domain in the previous section, we are now in a position to systematically move toward an embodied cognitive semantics. In this section, we turn to the question how the Talmyan schematic system perspective can be integrated into this embodied account. This is how Talmy introduces perspective:

The present schematic system consists of the perspective that one can have on [a referent] entity...this system thus establishes a conceptual perspective point [PP] from which the entity is cognitively regarded. While this schematic system is presumably neutral to particular sensory modalities, it is most readily characterized in visual terms as, in effect, pertaining to where one places one's "mental eyes" to "look out" upon a referent structure (2000a, p. 68).

Talmy has also shown that there can be a cognitive-semantic perspective point (PP) in both "space" and "time" (Talmy, 2000a), which corresponds to object spacetime and event spacetime in the framework of this paper (Section 2). In this section, I will show that Talmy's "mental eyes" actually "look out" via the physical eye, mostly into the peripersonal space right in front of us. In event spacetime, the physical eye looks upon the mental time line that we find in the (often peripersonal) space in front of our body.

As mentioned in the introduction, we will mainly use sentence (2)_Mary went to bed because she was tired - for exemplification purposes because this sentence involves (as so many sentences do) all the main schematic categories that Talmy proposes for cognitive semantics (Talmy, 2000a): configurational structure, attention, perspective (in both object spacetime and event spacetime), and force dynamics. Note that (2) clearly-albeit implicitly-contains temporal information: it suggests that Mary is first tired and then goes to bed. Stocker (2014b) analyzes this as a consecutive occurrence of cause and effect, as opposed to a simultaneous occurrence of cause and effect, which is the other possible temporal arrangement of cause and effect (cf. also Talmy 1988a, 2000a). As for (2), Mary will of course still be tired once she is in bed (at least before falling asleep), but the crucial aspect in relation to consecutive cause and effect is that the 'going to bed' only takes place as a subsequent event in relation to the onset of Mary's tiredness. (2) is a typical cause-effect conceptualization (called successful causation or s-causation in Stocker, 2014b): a cause prompts an effect, Mary's tiredness prompts her to go to bed.

We will assume that the speaker of sentence (2) relates an episodic (personal) memory: the speaker has actually seen Mary being tired and then going to bed. In terms of embodiment, it is important to become this specific about the temporal information-an issue that I am going to elaborate upon now before then returning to analyzing (2). There are three recent eye-tracking studies that for the (to the best of my knowledge) first time have shown that our eyes unconsciously look along a mentally construed time line while we cognitively process time. The first "ocular time line" finding relates to episodic memory and future thinking: our eyes look along a diagonal mental time line (left/down: past; right/up: future) while recalling an episodic memory image or imagining an episodic future image (Hartmann, Martarelli, Mast, \& Stocker, 2014). In this study of Hartmann and colleagues, participants where instructed to recall an 
episodic memory image or imagine an episodic future image for one minute (while looking at an empty screen in the critical condition). The left/down effect for the past and the right/up effect for the future was strongest after about 30-40 seconds-which is the time span that we know from another study that participants often need until they have a clear mental image in mind, when asked to generate one (D'Argembeau \& and Van der Linden 2004). The second "ocular time line" finding relates to serial recall: our eyes look along a left-to-right mental time line during recall tasks. When recalling (by speaking them out) serial items (five random digits), then the eyes look furthest to the left for the item that has been presented/encoded first - and the more recently the items have been presented/encoded, the more the eyes look to the right (Rinaldi, Brugger, Bockisch, Bertolini, \& Girelli, 2015). The third "ocular time line" finding relates to online auditory processing of temporal-relation words like before-that (German: vorher) and after-that (German: nachher). While listening to such words, the eyes look less upward when auditorily processing a before-that (past) relation, and more upward when processing an after-that (future) relation (within a time window of about 3500 milliseconds after the acoustic onset of the word) (Stocker, Hartmann, Martarelli, \& Mast, in revision). In this identified ocular time line, time is probably not only flowing upward but also forward at the same time (see Stocker et al., in revision for detailed argumentation). Taken together, these findings establish a first firm empirical basis that our eyes look along a mental time line while processing time, regardless of what kind of cognitive processing is involved (be it episodic memory/future thinking, working- memory serial recall, or online processing of temporal words). The possible functional status (as opposed to a merely epiphenomenal status) of eyes looking at or along a time line is discussed in Section 8.2.

Using the just discussed empirical findings (Hartmann et al., 2014; Rinaldi et al., 2015; Stocker et al., in revision) as a basis, I propose that the temporal-episodic information in relation to (2) is projected along a time line in peripersonal space in front of our body. As episodic processing suggests a left/down-to-up/right time line (Hartmann et al., 2014), I will provisionally assume that such a time line is involved when a speaker is processing a memory by uttering a statement like (2). As direct research on possibly existing time lines while verbally recollecting episodic memories is missing (we only have data for imagining episodic memories), it could also turn out that we conceptualize another time line during such a cognitive activity-for example, only left to right or no time line at all. However, given the current state of research (as just presented) I find it more likely that a mental time line is also mentally construed during verbally recalling episodic memories. Thus, such an assumption is built into Fig. 3. Whether this assumption really holds true, only future research can tell.

Stocker (2012a) provides a detailed analysis to demonstrate that the use of the past tense suggests that the past tense schematizes events on the time line as geometric points. As (2) also involves the past tense, the involved events can also be schematized as points on the spatialized time line in peripersonal space. In terms of cognitive semantics, the events belong to the principal member notion point of the schematic category degree of extension. This schematic category has three principal member notions (point, bounded extent, and unbounded extent) and belongs to the schematic system configurational structure (Talmy, 2000a, pp. 61-62).

We may also note that (2) involves chronological-order-reversed or antisequential processing of temporal information (Talmy, 2000a): the effect (Mary went to bed), which temporally takes place after the cause (she was tired), is linguistically evoked first (by being first mentioned). Cognitively processing the past tense within Mary went to bed is proposed to trigger off an eye 
gaze $\left(\mathrm{G}_{1}\right)$ that places event $\mathrm{B}$ (the effect) leftward/downward on the episodic mental time line in the peripersonal space in front of our body. Still cognitively processing the temporal aspect of because in because she was tired is proposed to then trigger off a second ocular gaze $\left(\mathrm{G}_{2}\right)$ that places event A (the cause) still more leftward/downward than event B on the episodic mental time line in the peripersonal space in front of our body.

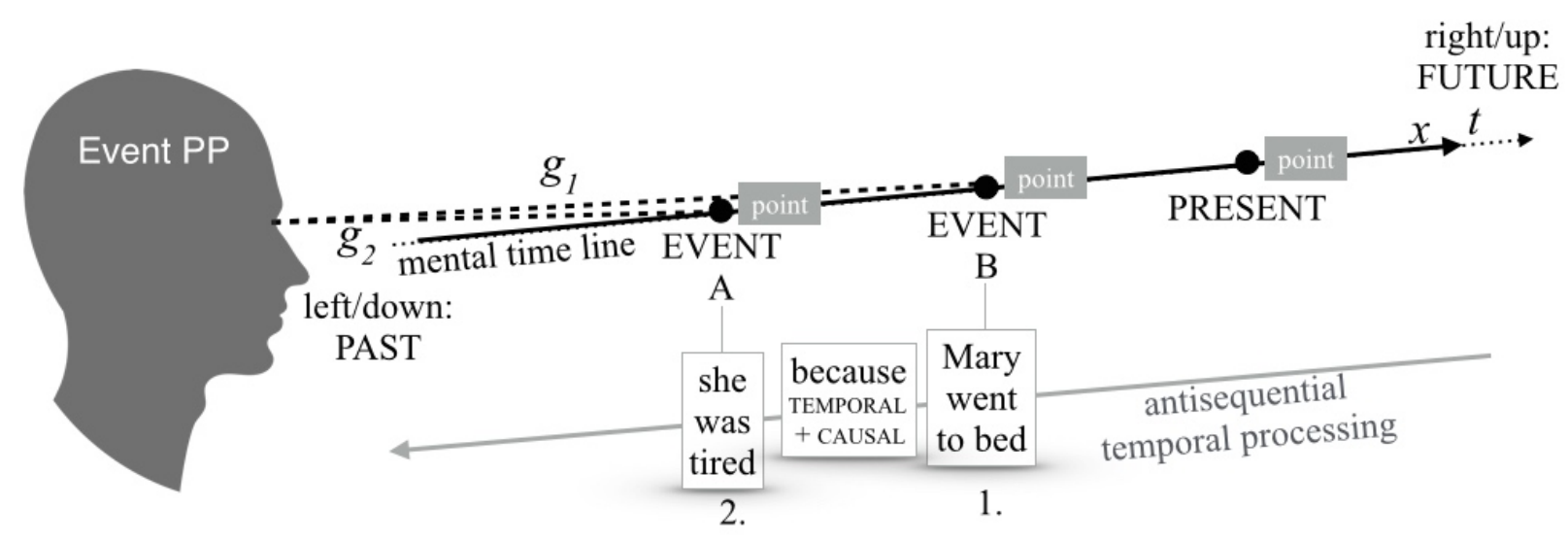

Figure 2: Cognitive-semantic perspective in "time" is developed into an ocular mental-time-line perspective in peripersonal space, using the temporal-ocular findings of Stocker and colleagues (in review) and Hartmann and colleagues (2014) as an empirical basis. Here, the episodic time line (past: left/down; future: right/up) is illustrated. Our eyes look along a diagonal mental time line (left/down: past; right/up: future) while processing episodic (personal) memory or episodic future thinking (Hartmann et al., 2014).

In this section, we have "ocularized" Talmy's cognitive-semantic system perspective in relation to the subdomain event spacetime (a foundational part of the domain cognitive spacetime, which in turn belongs to the schematic system configurational structure). Empirical evidence (Hartmann et al., 2014; Stocker et al., in revision) has been given that Talmy's "mental eyes," which "look out" (2000a, p. 68) on a time line (cf. Talmy, 2000, pp. 73-76), actually perform this "looking out" via the physical eyes. The eyes move along a mental time line that is mentally projected into the peripersonal space right in front of us.

\section{Embodied attention}

A foundational property of the Talmyan schematic system attention is the concept of Figure and Ground.

[There is a] pervasive system by which language establishes one concept as a reference point or anchor for another concept. It posits the existence in language of two fundamental cognitive functions, that of the Figure, performed by the concept that needs anchoring, and that of the Ground, performed by the concept that does the anchoring. The pair of concepts can be of two objects relating to each other in space...or the pair of concepts can be of two events relating to each other in a temporal, causal, or other type of situation (Talmy, 2000a, p. 311).

Talmy (2000a; 2007a) offers an elaborate attention as well as an elaborate Figure-Ground analysis. In this article, I will only focus on some of the basic aspects of the highly elaborate 
attention system: on some "definitional characteristics" and on some "typically associated characteristics" (Talmy, 2000a, pp. 315-316; 2007a) of Figure and Ground. Thus, this is only a first example of how cognitive- semantic attention phenomena can relate to ocular phenomena. The definitional characteristics of Figure and Ground are mainly concerned with positional and relational information. Given the embodied account of the episodic mental time line in the previous section - that the physical eye places the episodic mental time line in the peripersonal space in front of us with the past to the left and the future to the right-it follows naturally, where Figure and Ground occur in this embodied (ocularized) account: on the mental time line that is ocularly projected into peripersonal space. In relation to (2), the temporal location of the subordinate clause (because she was tired) serves as the temporal Ground, in relation to which the temporal location of the main clause (Mary went to bed) is defined (the content of the main clause thus functions as a temporal Figure). Moreover, the use of the past tense in (8) also fuses Mary went to bed (Figure) and she was tired (Ground) into a Composite Figure that, as a whole, is temporally located to yet another Ground (Ground of Composite Figure) (cf. Stocker, 2012a, pp. 399-400; Talmy, 2000a, pp. 336-337). This second Ground is the present moment because the past tense virtually by definition implies that the present moment is used as an anchor or reference point in relation to which the point in the past is temporally referred to (Comrie, 1985; Stocker, 2012a, 2014a; Talmy, 2000a).

One typical characteristic which is associated with Figure and Ground is that the "Figure is of greater relevance or concern than the Ground. The Figure is less immediately perceivable than the Ground but, once perceived, it is more salient, while the Ground is less salient once the Figure is perceived" (Talmy, 2007a, pp. XX; see also Talmy, 2000a, pp. 315-316). The greater relevance and salience of the Figure in relation to the Ground is also shown in the temporal (event-spatiotemporal) Figure and Ground along the ocular mental time line. For instance, in the already discussed study of Hartmann and colleagues (2014), participants looked to the left and downwards when retrieving an episodic memory image and to the right and upward when retrieving an episodic future image. Thus, the participants did not look at the temporal Ground (present moment), but at the temporal Figure (event in the past or future) while processing these memory images or future images (technically measured as mean eye gaze positions). This is just what one would expect if the Figure is of greater relevance and salience. These ocular findings then allow us to propose that the Figure and the Composite Figure in relation to (2) are more looked at by the physical eye (oculomotorically reflected attentional foregrounding) and that the Ground and the Ground of the Composite Figure are less (if it all) looked at by the physical eye (oculomotorically reflected attentional backgrounding).

Fig. 3 illustrates the proposed Figure and Ground manifestation on the episodic mental time line in the peripersonal space in front of the body. The thus far examined mental structure in relation to (2) — the mental time line and associated Figure and Ground assignments—remains invisible to the cognizer. For instance, in our study which showed that our eyes look along the episodic mental time line (Hartmann et al., 2014), the 19 participants (with one exception) remained unaware that they were looking along a mental time line. While the participants in our "ocular episodic time line" study (Hartmann et al., 2014) remained generally unaware of the mental time line, they were able to report what sort of episodic memory or episodic future images they experienced while locating the events on the time line. Thus images appear in relation to specific points on the ocular mental time line-which is the topic of the next section. 


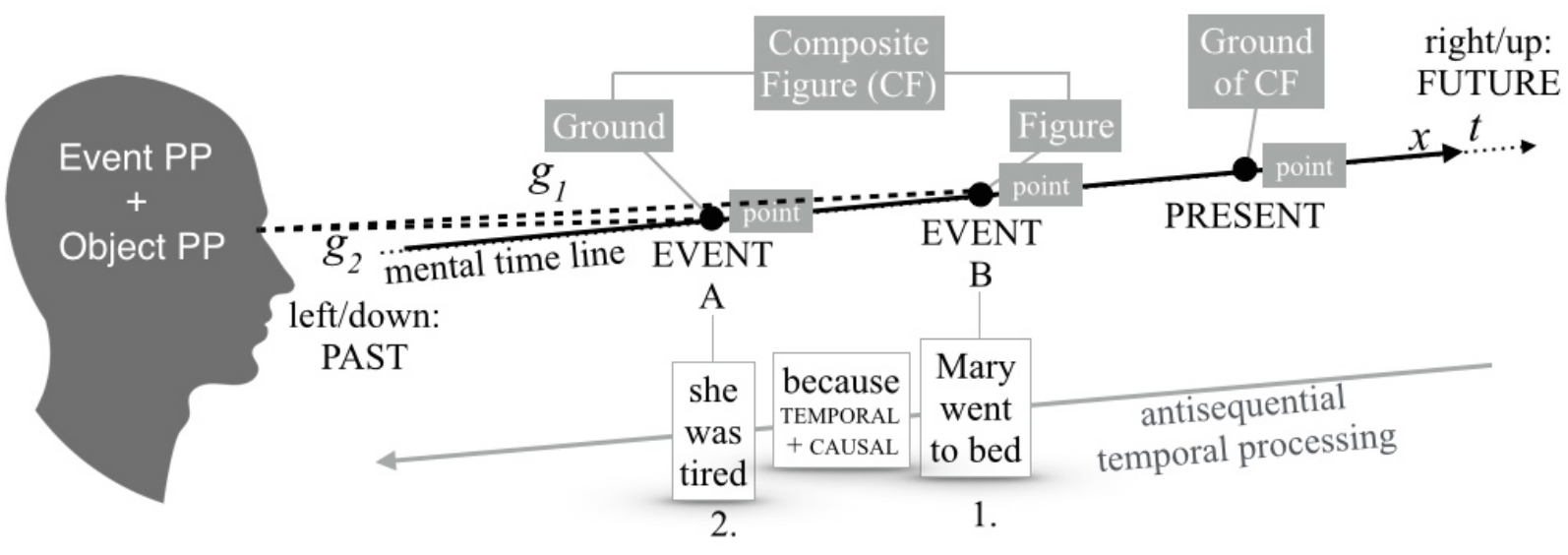

Figure 3: Cognitive-semantic temporal Figure and Ground (from the schematic system attention) are proposed to attach themselves to particular geometric points (event locations) on the ocular mental time line in peripersonal space. The event in the subordinate clause because she was tired serves as a temporal Ground, in relation to which the temporal location of the event in the main clause Mary went to bed is defined (temporally defined as the subsequent event). Moreover, temporal Figure (Mary went to bed) and temporal Ground (because she was tired) fuse to a Composite Figure. The present moment serves as the Ground of the Composite Figure, in relation to which the whole Composite Figure is put before the present moment, i.e., is put into the past.

In this section, we have added Talmy's schematic category Figure and Ground (part of the schematic system attention) to the subdomain event spacetime. Event-spatiotemporal Figure and Ground attach themselves to geometric points (cf. Section 2) on the ocular mental time line. Thus, empirical evidence (Hartmann et al., 2014) supports the notion that temporal Figure and Ground arrangements are also mentally projected into the peripersonal space right in front of us- onto the time line that our eyes project into this peripersonal space.

\section{Embodied perspective in "space"}

While Section 2 added embodied perspective in "time" (event spacetime), in this section we will now add embodied perspective in "space" (object spacetime) to the conceptual structure underlying (2).

At the mental-time-line location event $B$ (cf. Figs. 1-4), the unfolding of a corresponding mental image $i$ in the $x y z$-space of object spacetime could also take place while one is cognitively processing (2) - e.g., a mental image could emerge, in which the cognizer pictures Mary walking over to her bed, lying down on it. Similarly, the unfolding of a corresponding mental image $i$ in the $x y z$-space of object spacetime could then also take place at mental-time-line location event $A-$ e.g., $i$ in which Mary is cognized as looking tired and as not being in bed yet. In terms of ocular mind theory, the proposal depicted in Fig. 4 is that the physical eyes serve a double function. On the one hand (in event spacetime), the eye gaze is directed at the (invisible) locations Event A and Event B on the mental time line. On the other hand, the eyes also look out at the mental images that unfold in relation to these time-line locations. That mental images are 
also "acted out" by corresponding eye movements, even when people are only imagining or recalling such scenes, is well known (e.g., Johansson, Holsanova \& Holmqvist, 2006; Spivey \& Geng, 2001). In Fig. 4, the possible unfolding of a mental image is graphically illustrated by "image bubbles" that unfold in relation to the given time- line locations. Note also in Fig. 4 that the imagistic content within the $x y z$-space of object spacetime is represented schematically as the pictorial elements 'Mary', 'out of bed', 'tired' (at Event A) and 'Mary', 'goes to bed' (at Event B). Furthermore, the pictorial-locational information within Event A 'out of bed' is not explicitly expressed linguistically in (2). However, there is reason to believe that this implicit semantic information is mentally represented. Note that logically speaking, Mary must be out of bed during Event A; if she were in bed from the beginning, she could not still go there. The reason for the decomposition of the mental image into these proposed pictorial elements in Fig. 4 will become clear when, in the next section, we examine how force-dynamic elements attach themselves within a mental picture.

Finally we turn to the $t$ (time) of object spacetime. Fig. 4 represents a conglomerate structure of event spacetime (mental time line with ocularly indicated locations on it) and object spacetime (three-dimensional space with pictorial elements in it). In Fig. 4, the t of object spacetime (cf. Fig. 1a) is assumed to run along $x$ of event spacetime (along the mental time line), just as the $t$ of event spacetime does (cf. Fig 1b). Having one $t$ unfolding along event-spatiotemporal $x$ (rather than having two separate $t$ s running within a single mental scene) seems a plausible and also the most parsimonious solution for $t$ in mental representations that contain both object and event spacetime. The next section examines how configurational structure might manifest itself in relation to the pictorial elements in Fig. 4.

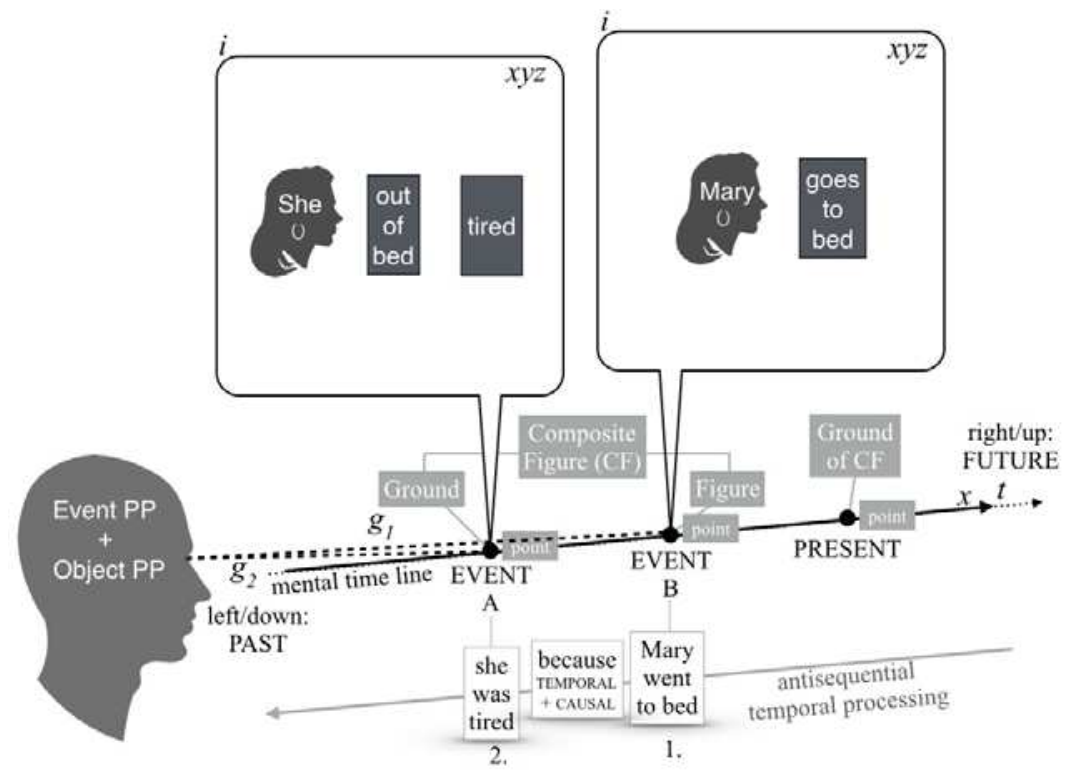

Figure 4: Cognitive-semantic perspective in "space" is developed into an ocular perspective. The participants of the study of Hartmann et al. (2014) looked along such a diagonal mental time line (left/down for the past and right/up for the future) while at the same time creating a mental image of an episodic past or possible episodic future event. The eyes thus serve a double function-looking at the mental time line in peripersonal space and looking at the mental image. That mental images are also accompanied by corresponding eye movements during imagining or recalling such scenes is well known (e.g., Johansson et al., 2006; Spivey \& Geng, 2001). 
In this section, we have "ocularized" Talmy's cognitive-semantic system perspective in relation to the subdomain object spacetime. Empirical evidence (e.g., Johansson et al., 2006; Spivey \& Geng, 2001) has been presented that has allowed us to interpret Talmy's "mental eyes" that "look out" (2000a, p. 68) at a spatial scene (cf. Talmy, 2000, pp. 68-69) as the physical eyes that project mental content into the peripersonal space in front of our body. Thus, the eyes serve a double function in cases_-like (2) — where both event spacetime and object spacetime are involved. The eyes look at a particular point (representing the location of an event) at the peripersonal mental time line and at the same time look out at the mental image that unfolds in relation to the time-line point.

\section{Some further aspects of embodied configurational structure in "space"}

We have already encountered the cognitive-semantic schematic system configurational structure in relation to the schematic categories domain and degree of extension in Section 2. In relation to domain, this has led to the introduction of the concept of cognitive spacetime with its two subdomains (domain member notions) object spacetime and event spacetime (Stocker, 2014c). In relation to degree of extension, it was proposed that the events on the mental time line are points (no extension) (Stocker, 2012a; Talmy, 2000a, p. 61). In this section we now turn specifically to more object- spatiotemporal configurational structure that can be identified in relation to (2) (cf. (4-C-2) and (4-F-2a-b)).

At its most general level, the Talmyan schematic system configurational structure involves

... the schematic structuring or geometric delineations in space [object spacetime] or time [event spacetime] or other qualitative domain that closed-class forms can specify. Closed- class forms can ascribe such structure to the whole of a referent scene, thus partioning that scene into entities in particular relationships, or to any of those entities themselves, or to the paths described by such entities when their interrelationships change through time (Talmy, 2000a, p. 47).

In (2)-Mary went to bed because she was tired - the open-class element Mary (from the openclass category proper name) already encodes the notion person as well as the subnotions uniplex and female (see (3-A)). The closed-class element she (pronoun) encodes the discourse-temporal information person last mentioned (anaphoric function) (4-G-2). Additionally, she also encodes (as the proper name Mary does) the notions uniplex and female. The closed-class element to furthermore specifies that Mary is moving toward an object. Thus, to delineates a path (cf. Talmy, 2000b, pp. 25-27). In the mental representation underlying (2), the path in essence extends from a location out of bed (cf. also Section 7) to the location bed.

Fig. 5 illustrates how the configurational elements uniplex and female are attached to the mentally represented person Mary. During Event A (which is the second event, due to antisequential processing) the configurational element last person mentioned projects back in time to establish to which mentally represented person she refers to (it refers to Mary, as she is the last person mentioned and as she carries the values uniplex and female). The configurational elements uniplex and female are proposed to occur in relation to the mental representation of Mary, which unfolds within the $x y z$-space of object spacetime in the space in front of our body. In contrast, the configurational element last person mentioned does not project through object spacetime, but along the mental time line (event spacetime) in the space in front of our body, as this 
element projects from event to event - from the speech event when "she" is uttered (Event A) to the event when Mary is first mentioned (Event B).

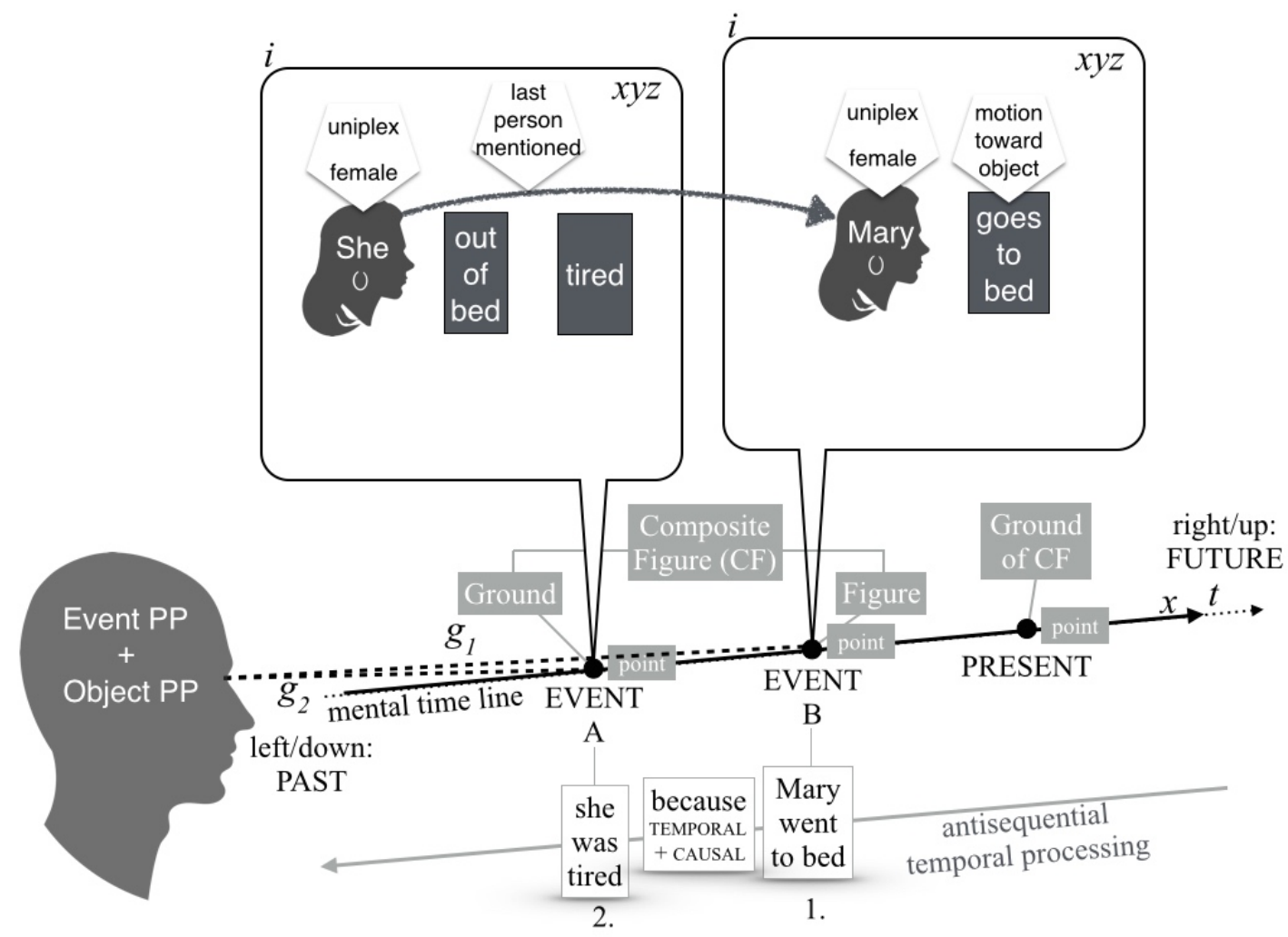

Figure 5: Cognitive-semantic configurational-structure elements in object spacetime (here, uniplex, female, and motion toward object) are proposed to structure mental images-here in relation to a mental image of Mary. Thus, configurational-structure elements attach to entities or their associated actions or states that occur within object spacetime. Additionally, the cognitive configurational- structure element last person mentioned - triggered off by the anaphoric component of she within Event A-projects along the mental time line of event spacetime to the moment when the referent is mentioned with a proper name ("Mary") in Event B.

In this section, we have added the member notions of the configurational-structure categories plexity (the notion uniplex), gender (the notion female), and path (the notion motion toward object) to entities or their associated states/actions within object spacetime. If cognizing these or other configurational structures are also reflected in the physical eye, has not been investigated yet. Section 8.2 discusses how complexity of configurational structure might be reflected in pupil size.

Furthermore, it has been shown that anaphoric reference in relation to persons (as for instance encoded in she) involves projecting the information last person mentioned along the mental time line, back to the moment where the referent's identity was established (for instance, by referring to a referent with a proper name such as Mary). If then the configurational object-spatiotemporal structure is identical between an entity and its anaphoric reference (Mary and she both configurationally encode uniplex and female), then the anaphoric reference can be successfully established. Given the theory of the ocular mind, all this configurational structure can be proposed to structure the conceptual content (Mary, goes, bed, tired) that unfolds in the spacetime in front of us. More precisely, one should perhaps no longer speak of "peripersonal 
space", but of "peripersonal spacetime." The embodied "home" of cognitive spacetime indeed seems, to a large extent, to be the space right in front of our body.

\section{Embodied force dynamics}

The Talmyan schematic system force dynamics involves

... how entities interact with respect to force. Included here is the exertion of force, resistance to such a force, the overcoming of such a resistance, blockage of the expression of force, removal of such blockage, and the like (Talmy, 2000a, p. 409).

The mental construction of cause and effect - the main concern of the current section-can also be systematically described in terms force dynamics. The force-dynamic system was first described by Talmy in 1985 (Talmy, 1985, 1988a, 2000a; see also Jackendoff, 1990, 1996; Pinker, 2007; Wolff, 2003, 2007). That there are force-dynamic patterns in our thinking has by now also been supported by experimental findings (Barbey \& Wolff, 2007; Wolff, 2003, 2007; Wolff \& Song, 2007). Stocker (2014b) has revised Talmy's force dynamics to some degree. With this revision it is now-among other aspects-for instance possible to apply force dynamics to causation in general, irrespective of how concrete or abstract the causal relationship is. In Talmy's account it was not always clear how force dynamics could be applied to certain abstract relationships such as cause-and-effect relationship between highly abstract ideational entities. This updated version of force dynamics is called elementary force dynamics (Stocker, 2014b) because this theory also offers an account of the conceptual primitives (force-dynamic elements) that are involved in mentally representing cause and effect.

Force dynamics, as proposed by Talmy (1985, 1988a, 2000a) and Stocker (2013; 2014b), involves the assumption that a mental representation of causality involves the mental representation of two conceptual entities that are in opposition and that are engaged in force interaction - for somewhat different views see Jackendoff (1996) and Wolff (2007). One of these two conceptual entities is called the agonist (Ago, different from the agonist in physiology). In Stocker's (2014b) revised version of force dynamics-in elementary force dynamics (Stocker, 2014b) - Ago is a mental entity that can be attributed any state or action (called value $\mathrm{x}$ ). The other of these two conceptual entities is called the antagonist (Ant, different from the antagonist in physiology). In elementary force dynamics, Ant is always conceptualized as wanting to impose a state or action onto Ago that is different from the initial action or state value that is associated with Ago (called value $\mathrm{x}_{\text {diff }}$, where diff stands for "different"). The resultant (effect) of a force-dynamic interaction is always formulated in relation to Ago. This outcome depends on which force (Ago's or Ant's force) is conceptualized as being stronger. The force-dynamic value stronger is signified with (+) and weaker with (-). In conceptualizations that can be phrased with because (of) - as in (2) —Ant is always stronger. With the just described basics of elementary force dynamics, we are now in a position to add force dynamic structure to (2) — to Mary went to bed because she was tired-whose conceptual structure has thus far been analyzed in terms of configurational structure, attention, and perspective (Sections 2-5).

In (2), Mary functions as Ago and Mary's tiredness as Ant (see Stocker, 2014b for a detailed analysis). In elementary force dynamic terms, Ago Mary is in the initial locational state out of bed. This is counteracted by her tiredness, which functions as Ant carrying the value 'indication to go to bed' (in the sense that Mary's tiredness suggests to Mary that it is indicated for her go to 
bed). In (2) Ant's suggestive force (indication to go to bed) is stronger than Ago's (Mary's) locational state of being out of bed. Hence Mary is conceptualized as having gone to bed because this is what her tiredness suggested to her. Of course, there might have been many intermediate steps between the onset of Mary's tiredness and her eventual going to bed. But (2) gaps all these intermediate steps, putting the attentional focus only on the initial tiredness and its end result of going to bed (cf. Talmy, 2000a, Chapter 4; 2007a).

Stocker (2014b) uses a specific notational system to capture force-dynamic interactions (an abstracted version of Talmy's original force-dynamic diagramming system). For a force-dynamic inter- action underlying our use of because-like (8) - the notational system looks as follows:

(7) $\mathrm{C}: \operatorname{Ago}-\mathrm{X}(-)$, Ant $^{-x_{\text {diff }}}(+) \rightarrow \mathrm{E}: \operatorname{Ago}_{\mathrm{diff}}$

The elementary force-dynamic structure of the sentence "Mary went to bed because she was tired" was analyzed in (Stocker, 2014b, p. 132):

(8) $\mathrm{C}: \mathrm{Ago}_{\underline{\text { Mary }}} \mathrm{X}_{\text {out of bed }}(-), \operatorname{Ant}_{\underline{\text { Mary's tiredness }}}-\mathrm{X}_{\text {diff indication to go to bed }}(+) \rightarrow \mathrm{E}:$ Ago $_{\underline{\text { Mary }}}-\mathrm{X}_{\text {diff goes to }}$ bed

The most natural descriptive solution to "add the [force-dynamic] forces that the elements of the [spatiotemporal] structural framework exert on each other" (Talmy, 2000a, p. 467) is to ascribe the corresponding force-dynamic elements to the mentally represented animate or inanimate entities (including abstract "entities" such as tiredness) and their associated actions/states that unfold within the $x y z$-space of object spacetime. The elements of the force-dynamic cause $C$ manifest at the event- spatiotemporal Event A and the elements of the force-dynamic effect $E$ at Event B. At $A$ and $B$ the $x y z t$-spacetime of object spacetime unfolds with a corresponding image within it (as described in Section 5). The force-dynamic elements attach to the mentally represented entities or to their associated action/states which unfold within this mental object spacetime. Thus, within the xyzt- spacetime unfolding at $A$ (cause), the force-dynamic element Ago is attached to the mental (e.g., imagistic) representation of Mary; the force-dynamic element action/state $x$ is attached to Mary's mentally (e.g., imagistically) represented location of being out of bed; and the force-dynamic element Ant is attached to the conceptualization of Mary's tiredness (her imagined physiological state). This conceptualized tiredness already projects into the future - to event B - that is, to the possibility that Mary's tiredness could cause her to go to bed. This projection into the future is represented in Fig. 6 with an arrow going from $\mathrm{x}_{\text {diff }}$ at $A$ to $\mathrm{x}_{\text {diff }}$ at $B$. It is the mental information goes to bed that attaches to the force-dynamic $\mathrm{x}_{\text {diff }}$ value. Then, within the $x y z t$-spacetime unfolding at $B$ (effect), the force-dynamic element Ago is still attached to the mental representation of Mary, but as Ant has by now successfully imposed its $\mathrm{x}_{\text {diff }}$ force onto Ago, the force-dynamic element $\mathrm{x}_{\text {diff }}$ value goes to bed is now attached to Mary. Since by the time that the effect has taken place, the $\mathrm{x}_{\text {diff }}$ value is already realized, Mary is indeed cognized as having gone to bed because she was tired.

Fig. 6 illustrates how the force-dynamic elements are attached to the mentally represented animate or inanimate entities (including abstract "entities" such as tiredness) and their associated actions/states that unfold within the $x y z$-space of object spacetime in the space in front of our body. 


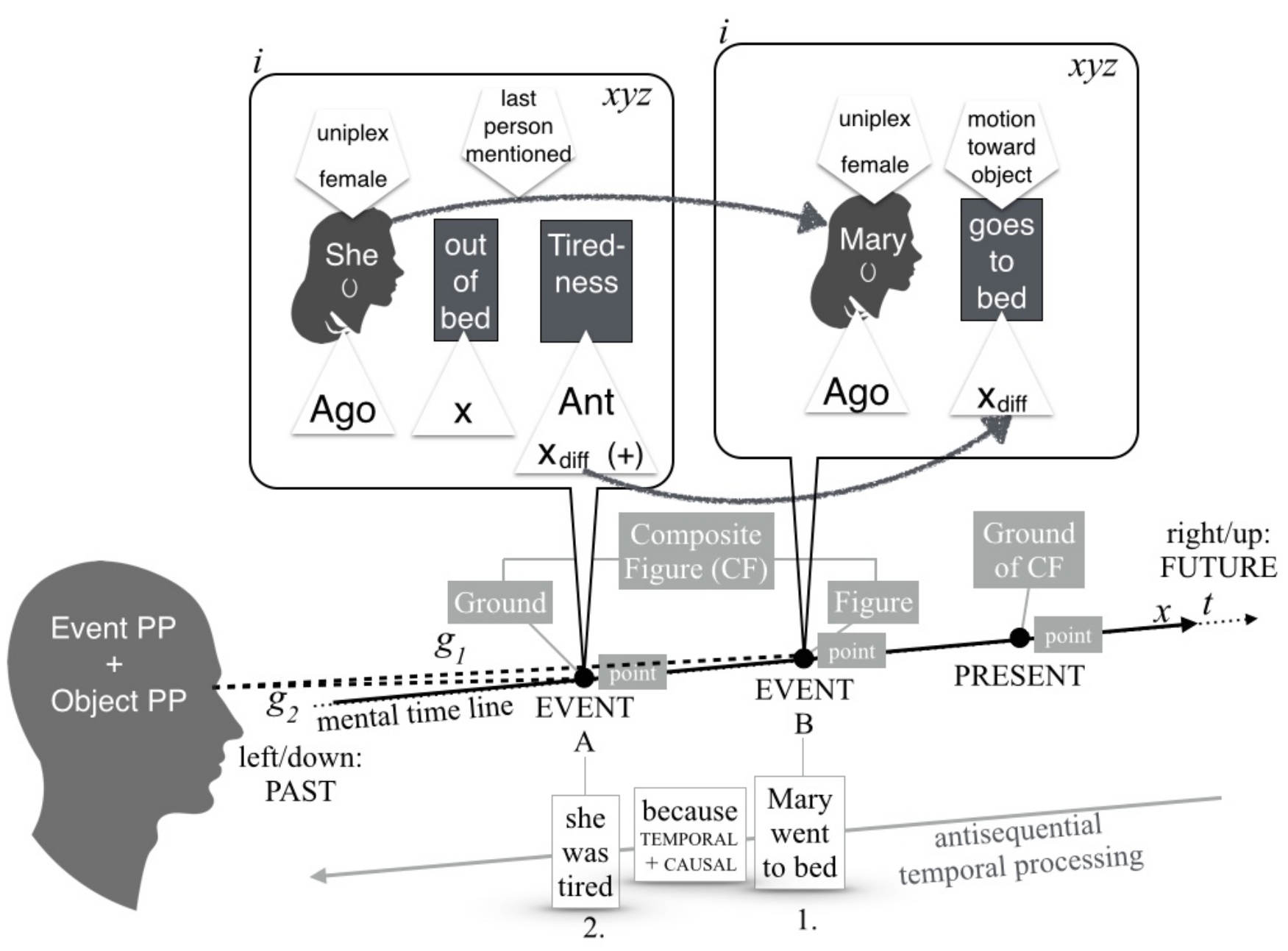

Figure 6: Cognitive-semantic force-dynamic interactions are proposed to occur within the object-spatiotemporal mental images that appear at particular event-spatiotemporal locations. Force-dynamic elements can in principle attach to the same entities and/or their associated action/states as configurational elements attach themselves to (cf. Section 6). Thus Ago attaches to Mary and Ant to the abstract entity Mary's tiredness. The indication of the force of the tiredness-its suggestion that it is time to go to bed-projects into the future (from event A to event B); thus it projects to the possibility that Mary's tiredness could cause her to go to bed. As Ant's force-indication to go to bed - is stronger than Mary's state out of bed, Mary is indeed conceptualized as going to bed (at the time of event B).

In this section, we have added force-dynamic elements to entities or their associated states/actions within the object-spatiotemporal mental images that appear in relation to particular points (event locations) on the mental time line. Moreover, as a final point worth stressing, we have seen that force-dynamic structure can also attach to content that is not explicitly expressed linguistically. For instance, Mary's (Ago's) initial x-value out of bed is not mentioned in (2) - in Mary went to bed because she was tired. This linguistically achieved gapping (Talmy, 2000a, Chapter 4; 2007a) is an indication that the image is more fully fleshed out than the linguistic expression thereof. Section 8.2 discusses how complexity of force dynamic structure might be reflected in pupil size. 


\section{Discussion}

In this article, I introduced an embodied account of cognitive semantics-more specifically, a theory of ocular cognitive semantics. The theory basically proposes an "ocular mind"- that thought is spatialized and that our eyes project this spatialized thought into the external (usually near) space in front of our body. This spatialized, "looked upon thought" can, as I hope to have been able to show, very fruitfully be described with the schematic systems of cognitive semantics-with configurational structure, attention, perspective, and force dynamics (Talmy, 2000a). Taking schizophrenia as a case in point, I will now discuss possible clinical implications of the theory of the ocular mind (or of ocular cognitive semantics) (Section 8.1). Then, finally, I will also discuss further empirical research which is needed for a more extended theory of embodied cognitive semantics (Section 8.2) before adding some concluding remarks.

\subsection{Clinical implications}

Discussing the implications of the theory of the ocular mind (of ocularized cognitive semantics), one could take the theory into many different realms. It could, I think, quite naturally be related to such areas as artificial intelligence, cognitive modeling, philosophy of mind, and computer science. Among the many options for which the implications of the theory of the ocular mind could be discussed, I chose a perhaps somewhat untypical one: clinical psychology. The choice might be untypical because it is, to my knowledge, not very common yet to relate findings from cognitive science-let alone cognitive linguistics or cognitive semantics- to clinicalpsychological fields such as psychiatry and psychotherapy. But why not? After all, much of cognitive science is devoted to gain an ever deeper understanding of the human mind. As clinical psychology reflects the phenomenon that many people have troubled minds, it seems quite natural to apply work which is devoted to trying to understand the mind to people that have trouble with it. I hope that one day in the not too distant future, the theory of the ocular mind can be used beneficially for such people. I will examine and discuss in what way this might be possible in relation to schizophrenia and ocular perspective in object spacetime. Schizophrenia is a severe mental disorder where people experience combinations of hallucinations, delusions, and disordered thinking and behavior.

There are two basic ways how we can see a mental image that involves ourselves (for instance a memory image): we either see the mental scene as if we are looking out of our own eyes (called field perspective or embodied mental gaze, EMG), or we are looking at ourselves from an external perspective and thus also see ourselves in the memory image (called observer perspective or disembodied mental gaze, DMG) (e.g., Stocker, 2012b).

It is not trivial through which of these perspectives we mentally see for instance a memory image. In a field memory perspective, one is known to retrieve richer accounts of affective reactions, physical sensations and psychological states, whereas in an observer memory perspective one is known to retrieve richer accounts of external (environmental) factorsdifferences that are also reflected in corresponding brain activity (Eich et al., 2009). A classic finding is also that more distant memories are more likely to be recalled from an observer perspective and more recent memories are more likely to be recalled from a field perspective (Nigro \& Neisser, 1983). 
Recently, the first study of field/observer perspective in patients with schizophrenia has shown that these patients recall their autobiographical memories significantly more often from an observer perspective when compared to healthy participants. Furthermore, patients with schizophrenia do not—unlike their healthy comparison participants—recollect more recent memories more from a field perspective (Potheegadoo et al., 2013). Potheegadoo and colleagues interpret the low frequency of field memory as reducing the likelihood that patients with schizophrenia regard themselves as the active agents of their own past: "The experiential sense of re-living personal events is thus diminished in patients with schizophrenia, giving rise to a passive and weakened sense of self" (p. 91).

To the best of my knowledge, the question whether looking at a mental image from a field or observer perspective might be reflected systematically in ocular correlates has never been addressed yet. However, a study by Rice and Rubin (2011) has uncovered a phenomenon that might well be reflected oculomotorically: in most observer memories (in healthy individuals), the mental scene is seen from eye level (as if the scene were straight ahead of one's eyes) or from above (as if one was looking down upon the scene). Eye-level or from-above observer memories make up about $80 \%$ of all observer memories and only about $20 \%$ of them are mentally seen from below (as if one was looking up upon the scene). The ocular mind theorythe proposal that seeing mental images or sensing abstract spatial mental scaffoldings are reflected in corresponding eye movements-makes clear predictions how the external vantage point (straight ahead, above, below) of observer memories might be reflected oculomotorically. First, looking straight ahead (eye level) at a mental scene should lead to corresponding "straightahead" (upward/downward-neutral) saccades; second, looking from above at a mental scene should lead to corresponding downward saccades; and finally, looking at a mental scene from below should lead to corresponding upward saccades. Accordingly, patients with schizophrenia might be more inclined to look straight ahead or downward with their eyes as they process their memories because they more often process their memories from an observer perspective (Potheegadoo et al., 2013). If future investigations were to find that patients with schizophrenia more often also oculomotorically look at their memories from eye level or from above (due to excessive use of the observer perspective), then a rehabilitation program, based on teaching patients with schizophrenia to relearn adopting a field perspective more often, could also be based on the ocular mind theory. Learning to retrieve their memories from a field perspective, these patients could for instance be encouraged to close their eyes (to minimize distracting visual input) while recollecting an autobiographical memory and to move their eyes as if they were in the midst of their memory and as if they were looking around at the mental scene from within their body. Such field-inducing exercises might eventually help to break through the observer-perspective fixation of these patients and help them to also see their memories from within their bodies again. As Potheegadoo and colleagues (2013) think, this might help these patients to regain the experience of a more active and stronger self. Fig. 7 illustrates the proposed observer-to-field intervention. These suggestions are of course speculations for a clinical application. While these ideas are based on empirical findings suggesting that mental perspective is reflected oculomotorically (e.g., Hartmann et al., 2014; Spivey \& Geng, 2001; Stocker et al., in revision), they are first and foremost research questions that would require careful investigation prior to their application in a therapeutic setting. 
(a)

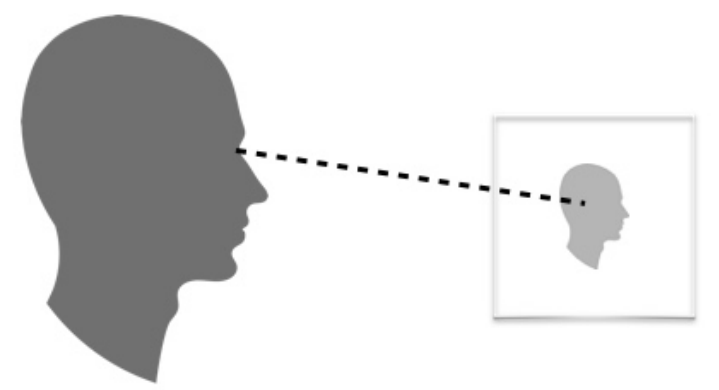

(b)

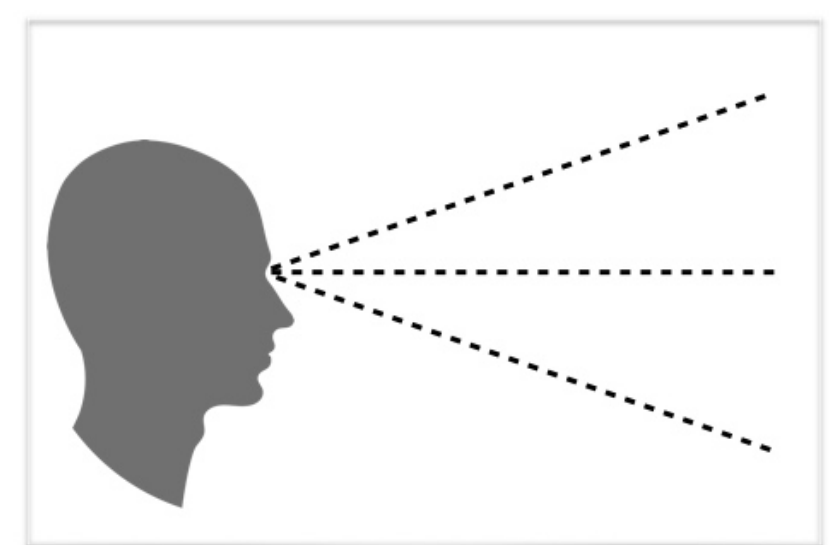

Figure 7: Ocular field and observer perspective and schizophrenia: a) According to the theory of the ocular mind, an observer perspective can be realized by using our eyes to project a mental image which includes oneself to the external (e.g., peripersonal) space in front of us. The observer mental image is symbolized by the picture frame. Note that the body with the eyes remains outside the mental picture; the location of the eyes represents this external vantage point. Oculomotorically, the theory of the ocular mind predicts that the observer perspective often involves looking straight ahead or downwards while retrieving the memory image, as observer memories are most often reported to be retrieved from these perspectives (Rice and Rubin, 2011). The study of Potheegadoo and colleagues (2013) suggests that patients with schizophrenia excessively externalize their self into such a body-exterior mental perspective as they recall their memories. b) According to the theory of the ocular mind, a field perspective can be realized by using our eyes to be in the midst of a mental image. Oculomotorically, the theory of the ocular mind predicts that the field perspective involves looking out of the eyes in any direction while processing the mental image, as the eyes are predicted to simply look at the scene in the same way as the scene was originally seen (which could also involve any direction). As described, patients with schizophrenia could during recall be taught to move their eyes as if they were in the midst of the memory (for instance with their eyes closed to minimize distracting visual input). Using the eyes in such a way might be helpful in inducing more frequent field perspective retrieval and thus in contributing to regaining a sense of a more active and a stronger self.

Akin to field and observer perspective, Talmy has also described how perspective points can involve looking out at a mental scene from within a mentally represented body or from outside a mentally represented body (cf. also Stocker, 2012b): 
Consider ... the following bit of narrative: "She sat in the rocker near her bed and looked out the window. How lovely the sky was!" In the first sentence, the use of a third-person pronoun together with the objective scene description invites the listener to place his perspective point somewhere in the depicted room looking at the sitting woman. But in the second sentence, the exclamatory how-construction, together with the expression of subjective experience, induces the listener to relocate his perspective point to the location of the sitting woman, in effect, looking out through her eyes (Talmy, 2000a, pp. 68-69).

As we can see in this quote, Talmy specifies which linguistic means are more likely to evoke an (observer-like) body-exterior perspective and which linguistic means are more likely to evoke a (field- like) body-interior perspective. It stands to reason that the linguistic means specified by Talmy (as well as further linguistic means) could be used as a complementary part in the treatment of patients with schizophrenia to induce more body-internal mental perspectives again. It also stands to reason that it would be useful to know how the physical eyes process such mental perspectives. This then is but one example how the worlds of embodied cognitive semantics and clinical psychology could meet.

\subsection{Further empirical research for embodied cognitive semantics}

In my view, the main question that ocular cognitive semantics faces is: does the gestural gaze which projects mental structure and content into peripersonal space merely play an epiphenomenal role or does it play a functional and facilitatory role in comprehending mental representations? There is at least one study which points to the possibility that the ocular time line might be functional in cognitively processing event spacetime. While it is well known that people with spatial neglect (with right-hemisphere lesions) neglect the left side of "space" (object spacetime), a new study has now also shown that these people additionally have difficulty with retrieving information in relation to past events (left side of mental time line), whereas they have no comparable difficulty with retrieving information in relation to future events (right side of mental time line) (Saj, Fuhrman, Vuilleumier, \& Boroditsky, 2014). Further functional confirmation for the theory of the ocular mind could come from interference studies. For instance: if people (in Western culture) were to perform a future- related task and at the same time had to look upward and/or rightward (congruent future task), would this assist future processing (for instance, faster reaction times in a future/past categorization task)? In contrast, if people were to perform a past-related task and at the same time had to look downward and/or leftward, would this facilitate processing the past (congruent past task)? In contrast, if people had to look upward and/or rightward while retrieving a past memory, would this incongruence interfere with past processing — and if people had to look downward and/or leftward while imagining the future, would this incongruence interfere with future processing? If so, we would have some first strong evidence for a functional status of ocular cognitive semantics in the realm of perspective in event spacetime. Comparable experimental interference paradigms could also be thought of in relation to perspective in object spacetime.

Experimental paradigms for the schematic systems configurational structure, attention, and force dynamics could be tested by cognitive effort paradigms. As we have seen, cognitive components like uniplex, female, motion toward object (configurational structure), Figure, Ground, Composite Figure, Ground of Composite Figure (attention), Ago, Ant, $x, x_{\text {diff }}$ etc. (force dynamics) can all attach to entities or their associated action/states in object spacetime (for instance in a mental image). It is also clear that not all concepts will have the same number of 
cognitive elements attached to them. For instance, the concepts cause and enable vary greatly in the number of force-dynamic elements attached to them (see Stocker, 2014b), with enable being force-dynamically much more complex than cause. In contrast, cause and despite share the exact same number of force-dynamic elements (it is just the force distribution that differs). Thus, processing concepts like cause and despite should involve less cognitive load than processing enable, as the latter involves processing more force-dynamic elements. Physiologically, greater cognitive load for instance shows in eliciting greater pupil dilation (Kahnemann \& Beatty, 1966) or in eliciting greater EEG-measured negativity (Münte, Schiltz, Kutas, 1998). Thus, processing fewer cognitive semantic elements versus processing more cognitive semantic elements in object-spatiotemporal configurational structure, attention, and force dynamics might be reflected in comparable physiological cognitive-load correlates.

\section{Conclusion}

This article has been called "Toward an embodied cognitive semantics." The concept toward is integrated because this article has exclusively dealt with the ocular aspect of embodied cognitive semantics. It has been shown how the schematic systems configurational structure, attention, perspective, and force dynamics can all be unified within an account of ocular cognitive semantics. However, a more comprehensive account of embodied cognitive semantics is likely to involve other embodied aspects than just the ocular one. Talmy (2015) for instance makes the case that force dynamics shares many cognitive-structural properties with somatosensory perception and motor control (pp. 25-27). In this newest article, Talmy fleshes out his "overlapping systems of cognitive organization" proposal quite comprehensively. His proposal is that different cognitive systems share structural properties to different degrees. Thus, he shows for example that the cognitive system language and the cognitive system visual perception have properties which they do not share (for instance the concept rotation features prominently in visual perception and only marginally in closed-class language), but also have some properties in common (for instance, possibly the way they structure space). Talmy stresses that

this outline [overlapping systems model of cognitive organization] is heuristic - its proposals about intracognitive relationships are solely suggestive - but it affords a framework that empirical approaches can amend or refine. Since my expertise is in language, all suggestions about other cognitive systems await others' expertise (Talmy, 2015, p. 2).

The present investigation has added one empirical piece to Talmy's model. It has been shown that the cognitive-semantic system perspective is not only structured by closed-class elements of language (Talmy, 2000a, 2015), but also by the physical eye that seems to project mental perspective right into the peripersonal space in front of our body.

\section{Acknowledgements}

I thank Thomas Fuyin Li as well as an anonymous reviewer for very thoughtful and helpful comments on earlier drafts of this manuscript. I also thank Ulrich Schnyder, head of the Department of Psychiatry and Psychotherapy at the University Hospital of Zurich, for having looked at and commented on my ocular intervention idea for schizophrenia. The work carried 
out in this article is part of my habilitation thesis at the University of Zurich (parts from the synopsis and outlook sections of the thesis).

\section{References}

Arzy, Shahar, Esther Adi-Japha, and Olaf Blanke. 2009. "The mental time line: An analogue of the mental number line in the mapping of life events." Consciousness and Cognition 18 (3): $781-785$.

Barbey, Aron K. and Phillip Wolff. 2007. "Learning causal structure from reasoning." In Proceedings of the 29th Annual Conference of the Cognitive Science Society, 713-718. Nashville, TN: Erlbaum.

Bergen, Benjamin and Kathryn Wheeler. 2009. "Grammatical aspect and mental simulation." Brain and Language 112: 150-158.

Casasanto, Daniel and Kyle Jasmin. 2012. "The hands of time: Temporal gestures in English speakers." Cognitive Linguistics 23 (4): 643-674.

Comrie, Bernard. 1985. Tense. Cambridge: Cambridge University Press. and Rafael. E. Núñez. 2009. "Across time, across the body: Transversal temporal gestures." Gesture 9 (2): 181-206.

D'Argembeau, Arnaud and Martial Van der Linden. 2004. "Phenomenal characteristics associated with projecting oneself back into the past and forward into the future: Influence of valence and temporal distance." Consciousness and Cognition 13 (4): 844-858.

Eich, Eric, Andrea L. Nelson, M. Adil Leghari, and Todd C. Handy. 2009. "Neural systems mediating field and observer memories." Neuropsychologia 47: 2239-2251.

Evans, Vyvyan. 2013. Language and Time: A Cognitive Linguistics Approach. Cambridge, England: Cambridge University Press.

Galton, Antony. 2010. "Time flies but space does not: Limits to the spatialisation of time." Journal of Pragmatics 43: 695-703.

Glenberg, A.M. and M.P. Kaschak. 2002. “Grounding language in action.” Psychonomic Bulletin \& Review 9 (3): 558.

Hartmann, Matthias, Corinna S Martarelli, Fred W Mast, and Kurt Stocker. 2014. "Eye movements during mental time travel follow a diagonal line." Consciousness and Cognition 30: 201-209.

Hartmann, Matthias and Fred W. Mast. 2012. "Moving along the mental time line influences the processing of future related words." Consciousness and Cognition 21: 1558-1562.

Huette, Stephanie, Bodo Winter, Teenie Matlock, David H Ardell, and Michael Spivey. 2014. 
"Eye movements during listening reveal spontaneous grammatical processing." Frontiers in Psychology 5: 1-7.

Ibarretxe Antuñano, Iraide. 2006. "Leonard Talmy. A windowing onto conceptual structure and lan- guage: Part 2: Language and cognition: Past and future." Annual Review of Cognitive Linguistics 4: 253-268.

Jackendoff, Ray. 1990. Semantic structures. Cambridge, MA: MIT Press.

Jackendoff, Ray. 1996. "Conceptual semantics and cognitive linguistics." Cognitive Linguistics 7 (1): 93-129.

Johansson, Roger, Jana Holsanova, and Kenneth Holmqvist. 2006. "Pictures and spoken descriptions elicit similar eye movements during mental imagery, both in light and in complete darkness." Cognitive Science 30 (6): 1053-1079.

Kahneman, Daniel and Jackson Beatty. 1966. "Pupil diameter and load on memory." Science 154 (3756): 1583-1585.

Kendon, Adam. 1967. "Some functions of gaze-direction in social interaction." Acta Psychologica 26: 22-63.

Knauff, Markus. 2013. Space to reason: A spatial theory of human thought. Cambridge, MA: MIT Press.

Lakoff, George and Mark Johnson. 1999. Philosophy in the flesh. New York: Basic Books. McNeill, David. 2006. “Gesture, gaze, and ground.” In Machine Learning for Multimodal Interaction, edited by Samy Bengio Steve Renals, 1-14. Springer.

Münte, Thomas F., Kolja Schiltz, and Marta Kutas. 1998. "When temporal terms belie conceptual order." Nature 395 (6697): 71-73.

Nigro, Georgia and Ulric Neisser. 1983. "Point of view in personal memories." Cognitive Psychology 15 (4): 467-482.

Núñez, Rafael. E. and Eve Sweetser. 2006. "With the future behind them: Convergent evidence from Aymara language and gesture in the crosslinguistic comparison of spatial construals of time." Cognitive Science 30 (3): 401-450.

Pinker, Steven. 2007. The stuff of thought: Language as a window into human nature. New York: Viking.

Potheegadoo, Jevita, Fabrice Berna, Christine Cuervo-Lombard, and Jean-Marie Danion. 2013. "Field visual perspective during autobiographical memory recall is less frequent among patients with schizophrenia." Schizophrenia Research 150 (1): 88-92.

Rice, Heather J. and David C.C. Rubin. 2011. "Remembering from any angle: The flexibility of visual perspective during retrieval.” Consciousness and Cognition 20 (3): 568-577. 
Rinaldi, L., P. Brugger, C. Bockisch, and L. Bertolini, G. Girelli. 2015. "Keeping an eye on serial order: Ocular movements bind space and time." Cognition 142: 291-298.

Saj, Arnaud, Orly Fuhrman, Patrik Vuilleumier, and Lera Boroditsky. 2014. "Patients with left spatial neglect also neglect the left side of time." Psychological Science 25 (1): 207-214.

Sinha, Chris, Vera da Silva Sinha, Jörg Zinken, and Wany Sampaio. 2011. "When time is not space: The social and linguistic construction of time intervals and temporal event relations in an Amazonian culture." Language and Cognition 3 (1): 137-169.

Spivey, Micheal J. and Joy J. Geng. 2001. "Oculomotor mechanisms activated by imagery and memory: Eye movements to absent objects." Psychological Research 65: 235-241.

Stocker, Kurt. 2012a. "The time machine in our mind." Cognitive Science 36 (3): 385-420.

Stocker, Kurt. 2012b. "Toward an embodiment-disembodiment taxonomy." Cognitive Processing - International Quarterly of Cognitive Science 13 (Suppl 1): S347-S350.

Stocker, Kurt. 2013. "Digital causal cognition.” International Journal of Cognitive Linguistics 4 (1): 9-34.

Stocker, Kurt. 2014a. "Mental perspective in multiple-event memory and foresight." Journal of Cognitive Science 15 (1): 57-95.

Stocker, Kurt. 2014b. “The elements of cause and effect." International Journal of Cognitive Linguistics 5 (2): 121-145.

Stocker, Kurt. 2014c. "The theory of cognitive spacetime." Metaphor and Symbol 29 (2): $71-$ 93. Stocker, Kurt, Matthias Hartmann, Corinna S. Martarelli, and Fred W. Mast. Under Review. "Eye movements reveal mental looking through time." Cognitive Science.

Talmy, Leonard. 1985. "Force dynamics in language and thought." In Papers from the twentyfirst regional meeting of the Chicago Linguistic Society, 293-337. Chicago Linguistic Society.

Talmy, Leonard. 1988a. "Force dynamics in language and cognition." Cognitive Science 12 (1): 49-100.

Talmy, Leonard. 1988b. "The relation of grammar to cognition." In Topics in cognitive linguistics, edited by Brygida Rudzka-Ostyn, 165-206. Amsterdam: John Benjamins.

Talmy, Leonard. 2000a. Toward a cognitive semantics. Vol. 1: Concept structuring systems. Cambridge, MA: MIT Press.

Talmy, Leonard. 2000b. Toward a cognitive semantics. Vol. 2: Typology and process in concept structuring. Cambridge, Mass.: MIT Press.

Talmy, Leonard. 2005. "The fundamental system of spatial schemas in language.” In From perception to meaning: Image schemas in cognitive linguistics, edited by Beate Hampe and Joseph E. Grady, Cognitive Linguistics Research 29, 199-234. Berlin/New York: Mouton de Gruyter. 
Talmy, Leonard. 2007a. "Attention Phenomena." In The Oxford handbook of cognitive linguistics, edited by Dirk Geeraerts and Hubert Cuyckens, 264-293. Oxford: Oxford University Press.

Talmy, Leonard. 2007b. "Foreword." In Methods in cognitive linguistics, edited by Monica Gonzalez- Marquez, Irene Mittelberg, Seana Coulson, and Micheal J. Spivey, Human Cognitive Processing 18. Amsterdam/Philadelphia: John Benjamins.

Talmy, Leonard. 2011. "Cognitive semantics: An overview." In Semantics: An international hand- book of natural language meaning, edited by Claudia Maierborn, Klaus von Heusinger, and Paul Portner, Vol. 1. De Gruyter Mouton.

Talmy, Leonard. 2015. "Relating language to other cognitive systems: An overview." Cognitive Semantics 1 (1): 1-44.

Ulrich, Rolf and Claudia Maienborn. 2010. "Left-right coding of past and future in language: The mental timeline during sentence processing." Cognition 117 (2): 126-138.

Wolff, Phillip. 2003. "Direct causation in the linguistic coding and individuation of causal events." Cognition 88 (1): 1-48.

Wolff, Phillip. 2007. "Representing causation." Journal of Experimental Psychology: General 136 (1): 82-111. 